\title{
Ginsenoside Rb1 inhibits hypoxia-induced epithelial-mesenchymal transition in ovarian cancer cells by regulating microRNA-25
}

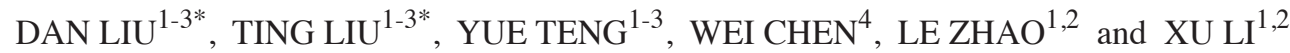 \\ ${ }^{1}$ Center for Translational Medicine; ${ }^{2}$ Key Laboratory for Tumor Precision Medicine of Shaanxi Province; \\ ${ }^{3}$ Department of Obstetrics and Gynecology; ${ }^{4}$ Center for Laboratory Medicine, The First Affiliated Hospital \\ of Xi'an Jiaotong University, Xi'an, Shaanxi 710061, P.R. China
}

Received September 22, 2016; Accepted May 19, 2017

DOI: $10.3892 / \mathrm{etm} .2017 .4889$

\begin{abstract}
Metastasis frequently occurs in advanced ovarian cancer, which not only leads to substantial mortality but also becomes a major challenge to effective treatment. Epithelial-mesenchymal transition (EMT) is a key mechanism facilitating cancer metastasis. Targeting the EMT process with more efficacious and less toxic agents to prevent metastasis is of significant therapeutic value for ovarian cancer treatment. The anti-EMT function and mechanism of ginsenoside Rb1, a monomer composition extracted from the traditional Chinese herb Panax ginseng or P. notoginseng, was investigated in the present study. Western blotting demonstrated that treatment with ginsenoside Rb1 antagonized hypoxia-induced E-cadherin downregulation and vimentin upregulation in SKOV3 and 3AO human ovarian cancer cells. Wound healing assays and in vitro migration assays indicated that ginsenoside Rb1 weakened hypoxia-enhanced cell migration ability. Furthermore, it was demonstrated that microRNA (miR)-25 is upregulated by hypoxia in ovarian cancer cells, which was attenuated by ginsenoside Rb1 treatment. Additionally, forced expression of miR-25 in ovarian cancer cells was identified to not only trigger EMT, but also block the suppressive effects of ginsenoside Rb1 on hypoxia-induced EMT by negatively targeting the E-cadherin transactivator, EP300. In conclusion, ginsenoside Rb1 may reverse hypoxia-induced EMT by abrogating the suppression of miR-25 on EP300 and E-cadherin, which suggests that ginsenoside Rb1 may be a potential therapeutic candidate for the treatment of ovarian cancer.
\end{abstract}

Correspondence to: Professor $\mathrm{Xu} \mathrm{Li}$ or Dr Le Zhao, Center for Translational Medicine, The First Affiliated Hospital of Xi'an Jiaotong University, 277 West Yanta Road, Xi'an, Shaanxi 710061, P.R. China

E-mail: lixu56@mail.xjtu.edu.cn

E-mail: zhaole2@mail.xjtu.edu.cn

*Contributed equally

Key words: ginsenoside, ovarian cancer, epithelial-mesenchymal transition, hypoxia, microRNA

\section{Introduction}

Ovarian cancer is the most lethal malignancy affecting the female reproductive system, with a 5-year survival rate as low as $30 \%$ (1). Cancerous metastasis is the leading cause of mortality in patients with ovarian cancer (2). The difficulty in treating disseminated cases constitutes a major hurdle to improve the prognosis of patients. Identifying agents with non-disclosed, anti-metastatic action from available therapeutics will be a short-cut strategy to combat cancer metastasis.

A growing body of evidence has demonstrated that loss of epithelial features and acquisition of mesenchymal phenotypes promote invasion and distant metastasis of cancer cells, the process is termed epithelial-mesenchymal transition (EMT) $(3,4)$. During EMT, loss of molecules involved in adhesion junctions, tight junctions and desmosomes cause disruption of cell-cell contact and cell polarity in epithelial cells, while acquisition of mesenchymal markers increases cell motility and invasion (5). EMT has been found to be positively implicated in metastatic development of various types of cancer (6). In ovarian cancer, EMT is reported to be associated with peritoneal metastasis, and poor progression-free and overall survival (7). Therefore, identification of agents that block the EMT process may increase the arsenal for treatment of ovarian cancer.

MicroRNA (miR) are a class of small non-coding RNA of $\sim 22$ nucleotides in length, that are single-stranded and highly conservative in structure. $\mathrm{miR}$ inhibit protein translation or promote mRNA degradation by binding to the 3 -untranslated region of their target genes (8). miR may act as oncogenes or tumor suppressors, depending on the specific function of their targets (9). Research has demonstrated the aberrant expression of miR in human ovarian cancer. For instance, miR-187 is overexpressed in ovarian cancer tissues, which is correlated with the overall survival of ovarian cancer patients (10). miR-149, a member of the miR-200 family, is capable of reversing the EMT process in ovarian cancer cells (11). miR-25, a member of the miR-106-25 cluster, located in intron 13 of the mini-chromosome maintenance complex component 7 on chromosome $7 q 22.1(12,13)$, is upregulated in epithelial ovarian cancer tissues and cell lines (14). Higher level of miR-25 present in tissues of advanced clinical stage and lymph node metastases of ovarian cancer, and patients with upregulated miR-25 tend 
to have poorer prognoses (15). However, the specific role of miR-25 in ovarian cancer metastasis remains unclear.

Several monomer compositions from traditional Chinese medicine possess anti-EMT activity. Resveratrol, for example, reverses epidermal growth factor-induced EMT via targeting the extracellular signal-regulated kinase signaling pathway in human breast cancer cell line MCF-7 (16). Ginsenosides are the pharmacologically active components of Panax ginseng $(17,18)$. Several effective ingredients of ginsenosides have been identified, such as Rb1, Rg1, Rg3, Rh1 and Rh2, the majority of which have been observed with anticancer activities (18-21). Rb1 has been found to have antioxidant, anti-inflammatory (22), anti-senescent and neural protective functions (23). The anti-neoplastic action of Rb1 has been reported in the SW480 human colorectal cancer cell line, where it may induce cell apoptosis (24). In the present study, the effect of ginsenoside Rb1 on hypoxia-induced EMT in SKOV3 and $3 \mathrm{AO}$ ovarian cancer cells was explored, attempting to elucidate the anticancer mechanism of Rb1 at the molecular level.

\section{Materials and methods}

Drug and antibodies. Ginsenoside Rb1 with purity of $99 \%$ was obtained from Tasly Pharmaceutical Co. (Tianjin, China). $\mathrm{Rb} 1$ was dissolved in dimethyl sulfoxide at a concentration of $1 \mathrm{mg} / \mathrm{ml}$ and stored at $-20^{\circ} \mathrm{C}$. The stock solution was then added into cell culture medium to generate certain final concentrations. The following primary antibodies were used: $\beta$-actin and vimentin (both from Cell Signaling Technology, Inc., Danvers, MA, USA); E-cadherin (Bioworld Technology, Inc., St. Louis Park, MN, USA); and EP300 (Sigma-Aldrich; Merck KGaA, Darmstadt, Germany).

Cell culture and treatment. Human ovarian cancer cell lines, SKOV3 and 3AO, were obtained from the Shanghai Cell Bank of Chinese Academy of Sciences (Shanghai, China) and the Shandong Academy of Medical Sciences (Jinan, China), respectively. Cells were cultured in RPMI-1640 medium supplemented with $10 \%$ (v/v) fetal bovine serum (FBS) (both from Gibco; Thermo Fisher Scientific, Inc., Waltham, MA, USA) . Control cells were cultured under normoxic conditions and maintained in an incubator at $5 \% \mathrm{CO}_{2}, 37^{\circ} \mathrm{C}$ and $100 \%$ humidity. For hypoxic induction, cells were incubated at $1 \% \mathrm{O}_{2}$ in a HF100 hypoxia chamber (Heal Force, Hong Kong, China) with or without exposure to $160 \mu \mathrm{g} / \mathrm{ml} \mathrm{Rb1}$. Cell morphology was observed and cell images were captured using a phase contrast microscope at a magnification of x 100 .

Cell viability assay. SKOV3 and $3 \mathrm{AO}$ ovarian cancer cells were seeded in 96-well plates at a density of $5 \times 10^{3}$ cells/well. Rb1 was used at final concentrations of $0-320 \mu \mathrm{g} / \mathrm{ml}$ to treat cells at $37^{\circ} \mathrm{C}$ for 24,48 and $72 \mathrm{~h}$, respectively. Cell viability was assessed by MTT colorimetric assay, with $20 \mu \mathrm{l}$ of $5 \mathrm{mg} / \mathrm{ml}$ MTT added into each well. This was followed by incubation with cells for $4 \mathrm{~h}$ at $37^{\circ} \mathrm{C}$ and the addition of $150 \mu \mathrm{DMSO}$, as previously described (25). The absorbance was measured at a wavelength of $570 \mathrm{~nm}$ by an EnSpire multimode plate reader (PerkinElmer, Inc., Waltham, MA, USA). The survival rate of cells was calculated as: Survival rate $(\%)=$ optical density
(OD) 570test - OD570blank]/(OD570control - OD570blank) $\mathrm{x} 100 \%$.

miR mimic transfection. SKOV3 cells were seeded, at a density of $2.5 \times 10^{5}$ cells/well into 6 -well plates $24 \mathrm{~h}$ prior to the transfection. A total of $100 \mathrm{nM}$ miR-25 mimic was provided by Guangzhou RiboBio Co., Ltd. (cat. no. miR10000081-1-5; Guangzhou, China) and transfected into cells in each well using the X-tremeGENE siRNA transfection reagent (Roche Molecular Diagnostics, Pleasanton, CA, USA), according to the manufacturer's instructions. A negative control was used in parallel experiments. After 24 or $48 \mathrm{~h}$ of transfection, total RNA and protein were collected, respectively.

Reverse transcription-quantitative polymerase chain reaction (RT-qPCR). Total RNA was extracted from treated SKOV3 and $3 \mathrm{AO}$ cells and corresponding control cells using TRIzol reagent (Invitrogen; Thermo Fisher Scientific, Inc.), according to the manufacturer's protocol. miR-25 was quantified by RT-qPCR. cDNA synthesis was conducted with RevertAid first strand cDNA synthesis kit (Thermo Fisher Scientific, Inc.; cat. no. K1622) according to the manufacturer's instructions, as follows: $2 \mu \mathrm{g}$ RNA was mixed with $1 \mu \mathrm{l}$ stem-loop reverse transcription primers for miR-25 (cat. no. miRQ0000081-1-1) and U6 (cat. no. MQP-0201; Guangzhou RiboBio Co., Ltd.) in a total volume of $12 \mu \mathrm{l}$, and incubated at $65^{\circ} \mathrm{C}$ for $5 \mathrm{~min}$ followed by being chilled on ice for $5 \mathrm{~min}$, then mixed with $4 \mu \mathrm{l} 5 \mathrm{x}$ reaction buffer, $2 \mu \mathrm{l}$ dNTP mixture, $1 \mu \mathrm{l}$ RNase Inhibitor and $1 \mu \mathrm{l}$ M-MuLV reverse transcriptase in a final volume of $20 \mu \mathrm{l}$. The reactions were performed at $25^{\circ} \mathrm{C}$ for $5 \mathrm{~min}$, followed by $42^{\circ} \mathrm{C}$ for $60 \mathrm{~min}$ and $70^{\circ} \mathrm{C}$ for $5 \mathrm{~min}$. The cDNAs were stored at $-80^{\circ} \mathrm{C}$ for subsequent assessments. qPCR was conducted on a CFX-96 real-time PCR system (Bio-Rad Laboratories, Inc., Hercules, CA, USA) using a SYBR-Green Master Mix (Takara Biotechnology Co., Ltd., Dalian, China). The reactions were incubated at $95^{\circ} \mathrm{C}$ for $30 \mathrm{sec}$, followed by 40 cycles of $95^{\circ} \mathrm{C}$ for $5 \mathrm{sec}, 60^{\circ} \mathrm{C}$ for $30 \mathrm{sec}$ and $72^{\circ} \mathrm{C}$ for $30 \mathrm{sec}$. Each measurement was performed in triplicate, and no-template controls were included for each assay. Small nuclear RNA (Rnu6; Guangzhou RiboBio; cat. no. MQP-0201) was used as an internal control. The relative expression of miR-25 was calculated using the $2^{-\Delta \Delta \mathrm{Cq}}$ method (26), normalized with the internal control and compared with the level in the negative control cells.

Western blot analysis. Total cell protein extracts were collected using mammalian protein extraction reagent (Pierce; Thermo Fisher Scientific, Inc., Waltham, MA, USA). The samples were quantified using a Quick Start Bradford Protein Assay kit (Bio-Rad Laboratories, Inc.). Proteins were boiled for $5 \mathrm{~min}$ prior to separation by $10 \%$ SDS-PAGE with $30 \mu \mathrm{g}$ proteins loaded in each lane, and then transferred onto nitrocellulose membranes (Pall Life Sciences, Port Washington, NY, USA). Membranes were blocked with 5\% non-fat milk at room temperature for $2 \mathrm{~h}$. The membranes were incubated with corresponding primary antibodies: $\beta$-actin (catalogue no. 3700; Cell Signaling Technology, Danvers, MA, USA), vimentin (cat. no. 5741; Cell Signaling Technology, Inc.), E-cadherin (cat. no. BS1098; Bioworld Technology, Inc., St. Louis Park, MN, USA) and anti-EP300 (cat. no. SAB1400094; Sigma-Aldrich; Merck KGaA) diluted in Tris-buffered saline-Tween-20 

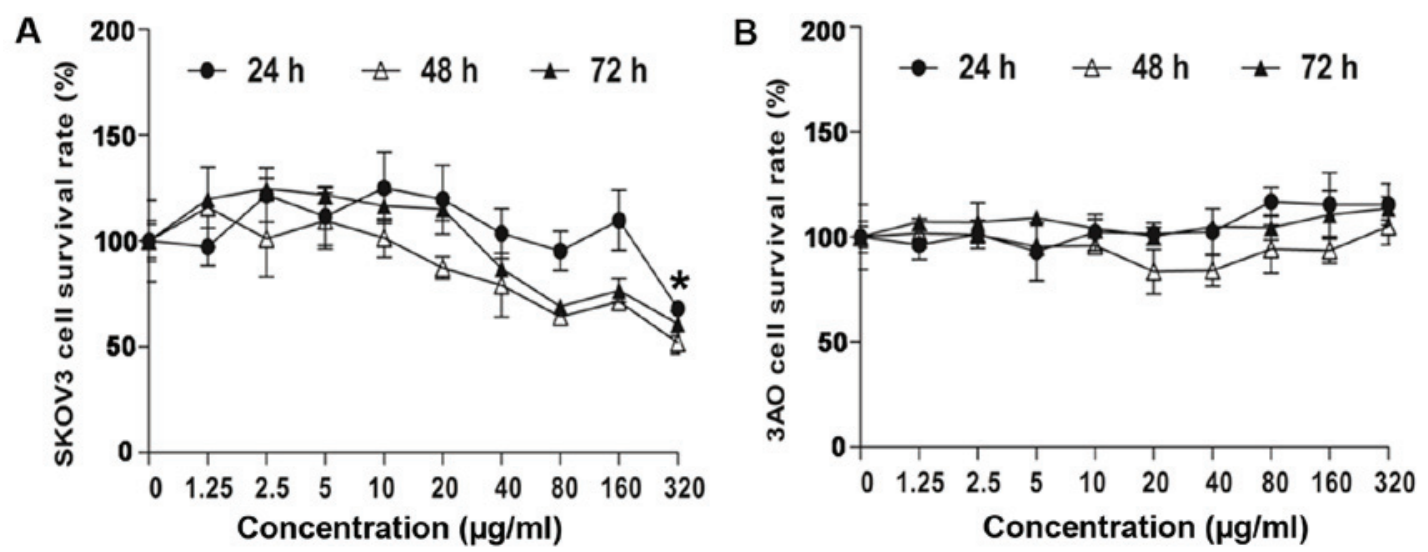

Figure 1. Effect of ginsenoside Rb1 on SKOV3 and 3AO ovarian cancer cell viability. (A) Effect of Rb1 at various doses and durations on cell viability of SKOV3 cells. Concentrations $<320 \mu \mathrm{g} / \mathrm{ml} \mathrm{Rb1}$ had no influence on SKOV3 cell viability at the indicated time points. However, for $24-\mathrm{h}$ incubation, $320 \mu \mathrm{g} / \mathrm{ml}$ $\mathrm{Rb} 1$ significantly reduced cell viability compared with all other concentrations (" $\mathrm{P}<0.05$, two-tailed t-test). (B) Effect of Rb1 at various doses and durations on cell viability of $3 \mathrm{AO}$ cells. All chosen doses of Rb1 at different durations demonstrated no influence on cell viability of $3 \mathrm{AO}$ cells.

(TBST), as follows: E-cadherin (1:500); vimentin (1:2,000); $\beta$-actin (1:1,000); and EP300 (1:500), respectively. Following three washes for $10 \mathrm{~min}$ in TBST, the membranes were incubated with the following horseradish peroxidase-conjugated secondary antibodies: Goat anti-mouse immunoglobulin (IgG; cat. no. 31430) and goat anti-rabbit IgG (cat. no. 31460; Thermo Fisher Scientific, Inc.) at a dilution of 1:2,000. Enhanced chemiluminescent reagent (Santa Cruz Biotechnology, Inc., Dallas, TX, USA) was used to develop the blots. $\beta$-actin was used as a loading control.

Wound healing assay. Cells were seeded into 6-well plates at a density of $4 \times 10^{5}$ cells/well for 24 h to reach $95 \%$ confluence as a monolayer. One scratch was created in each well using a sterile 200- $\mu$ l pipette tip. Cells were then cultured at $5 \% \mathrm{CO}_{2}$, $37^{\circ} \mathrm{C}$ for $24 \mathrm{~h}$ in serum-free medium. The process of wound healing was photographed at different time points under a phase-contrast microscope at a magnification of $\times 100$.

Cell migration assay. Treated- and control SKOV3 and 3AO cells $\left(1 \times 10^{5}\right.$ cells/well) in $100 \mu \mathrm{l}$ media without serum were added into Millicells with a pore size of $8 \mu \mathrm{m}$ (EMD Millipore, Billerica, MA, USA) and inserted into wells containing RPMI-1640 with 20\% FBS as a chemotactic factor. After $24 \mathrm{~h}$ incubation at $5 \% \mathrm{CO}_{2}$ and $37^{\circ} \mathrm{C}$, the cells remaining on the upper surface of the filter were removed with a cotton swab, and the migrating cells were fixed with $100 \%$ methanol at $4^{\circ} \mathrm{C}$ for $30 \mathrm{~min}$ followed by Giemsa staining at room temperature for $30 \mathrm{~min}$ for quantitation of cell number. The numbers of migrating cells in three high power fields on the lower surfaces of the membranes were counted under a phase-contrast microscope at a magnification of x100. A minimum of three wells were counted per experiment.

Statistical analysis. Data were presented as the mean \pm standard deviation. Statistical analysis was performed using SPSS 19.0 (IBM Corp., Armonk, NY, USA) and GraphPad Prism 5 software (GraphPad Software, Inc., La Jolla, CA, USA). Statistical differences between two groups were determined by Student's t-tests. $\mathrm{P}<0.05$ was considered to indicate a statistically significant difference.

\section{Results}

Effect of Rb1 on viability of SKOV3 and $3 A O$ ovarian cancer cells. MTT assays were used to examine the effect of ginsenoside Rb1 on the viability of SKOV3 and 3AO cells. SKOV3 or $3 \mathrm{AO}$ cells were incubated with ginsenoside $\mathrm{Rb} 1$ at various doses ranging from $0-320 \mu \mathrm{g} / \mathrm{ml}$ for 24,48 and $72 \mathrm{~h}$, respectively. As demonstrated in Fig. 1A and B, Rb1 only weakly affected the growth of SKOV3 and 3AO cells. Specifically, viability of SKOV3 cells was insignificantly affected by Rb1 treatment for $24 \mathrm{~h}$, with the exception of the maximum concentration, which demonstrated significantly impaired cell viability compared with all the other concentrations at $24 \mathrm{~h}(\mathrm{P}<0.05)$, while survival of SKOV3 cells was slightly suppressed by longer treatment (48 and $72 \mathrm{~h}$ ) of Rb1 at concentrations higher than $40 \mu \mathrm{g} / \mathrm{ml}$. As for $3 \mathrm{AO}$ cells, all doses of Rb1 demonstrated no significant influence on cell viability (Fig. 1B). As a 24-h incubation with $160 \mu \mathrm{g} / \mathrm{ml}$ of Rb1 had little effect on the viability of both cell lines, cells were treated with $160 \mu \mathrm{g} / \mathrm{ml} \mathrm{Rb1}$ for $24 \mathrm{~h}$ to perform the subsequent experiments.

Rbl blocks hypoxia-induced EMT in SKOV3 and $3 A O$ cells. As demonstrated in Fig. 2, when exposed to $1 \% \mathrm{O}_{2}$ hypoxic conditions for $24 \mathrm{~h}$, both SKOV3 and $3 \mathrm{AO}$ cells experienced typical EMT changes. Morphologically, both cell lines changed from an epithelial appearance of a cobblestone-like cellular shape and tight intracellular connections to a mesenchymal appearance of a spindle-like cellular shape and loose intracellular connections (Fig. 2A). When incubated with $160 \mu \mathrm{g} / \mathrm{ml} \mathrm{Rb1}$ under hypoxic conditions, cell morphology and cell junctions recovered to the epithelial pattern (Fig. 2A). Key EMT markers were further determined by western blot analysis. After hypoxic incubation for $24 \mathrm{~h}$, the expression of E-cadherin was decreased and the expression of vimentin was increased (Fig. 2B). Similar to the morphological observation, co-treatment with Rb1 under hypoxic conditions rescued E-cadherin downregulation and vimentin upregulation (Fig. 2B).

Additionally, cell mobility was detected by wound healing and in vitro migration assays. As demonstrated in Fig. 3A and $\mathrm{B}$, the wound healing ability of SKOV3 and $3 \mathrm{AO}$ cells 
A
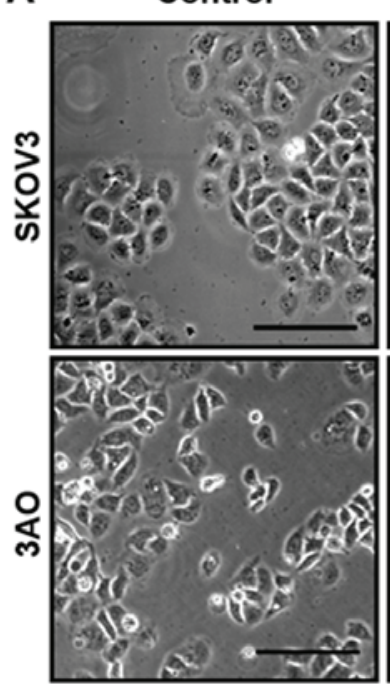

Ho24h+Rb1

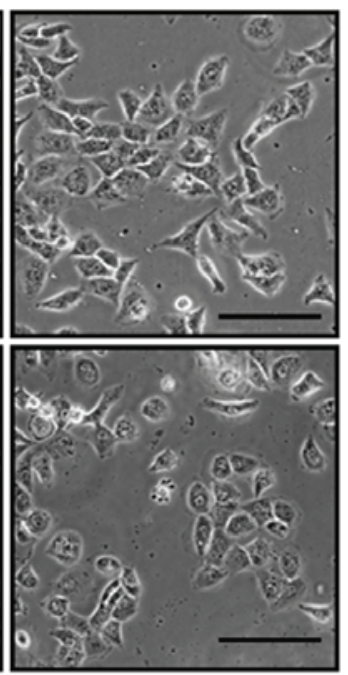

B
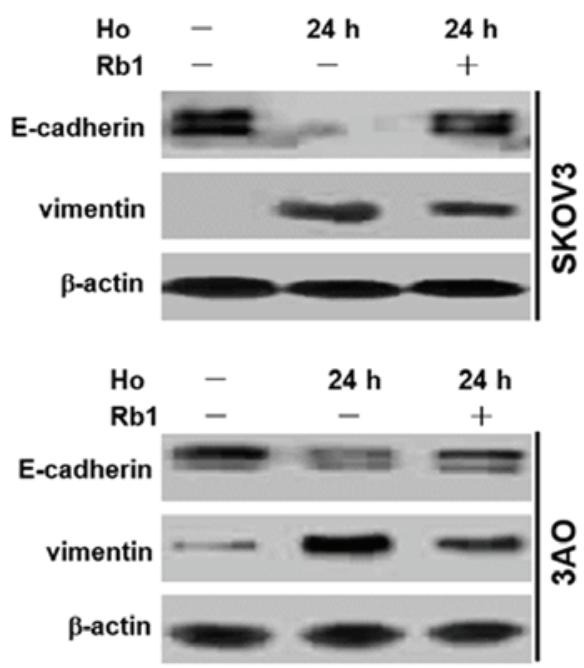

Figure 2. Ginsenoside Rb1 blocks the Ho-induced EMT process in SKOV3 and 3AO ovarian cancer cells. (A) Rb1 reversed the morphological EMT changes induced by $\mathrm{Ho}$ for $24 \mathrm{~h}$ in SKOV3 and $3 \mathrm{AO}$ cells. When exposed to $1 \% \mathrm{O}_{2}$ Ho for $24 \mathrm{~h}$, both cell lines changed from a polygonal, cobblestone-like cellular shape with tight intracellular connections to a spindle-like cellular shape with loose intracellular connections. When co-incubated with $160 \mu \mathrm{g} / \mathrm{ml} \mathrm{Rb} 1$, the morphology and cell junctions of both SKOV3 and 3AO cells recovered to an epithelial pattern. Scale bar, $100 \mu \mathrm{m}$. (B) Rb1 antagonized the Ho-induced expression alterations of EMT markers. Western blot analysis demonstrated Ho 24 h-incubation decreased the expression of E-cadherin and increased the expression of vimentin, which was recovered by $\mathrm{Rb} 1$ treatment under the $1 \% \mathrm{O}_{2}$ condition. EMT, epithelial-mesenchymal transition; Ho, hypoxic.

A

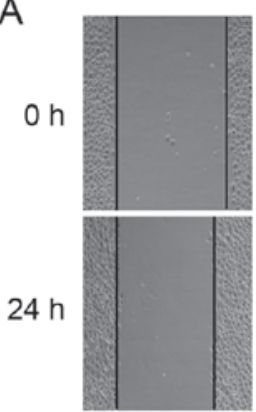

Control

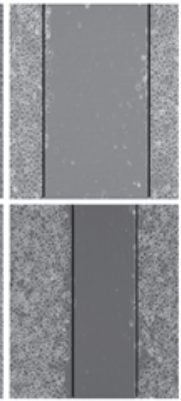

Hypoxia

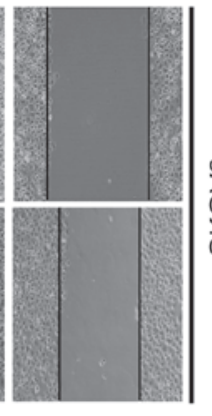

Hypoxia+Rb1

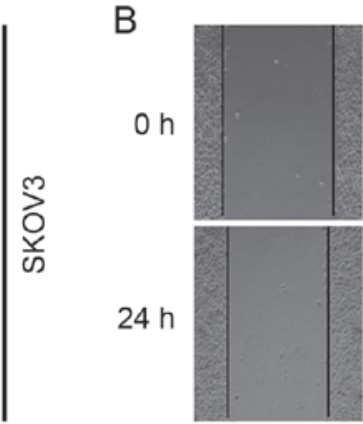

Control

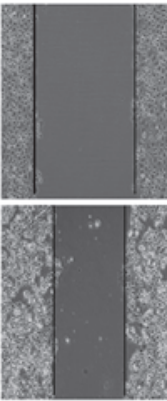

Hypoxia

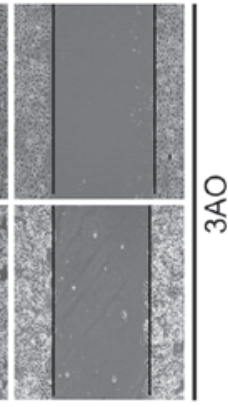

Hypoxia+Rb1
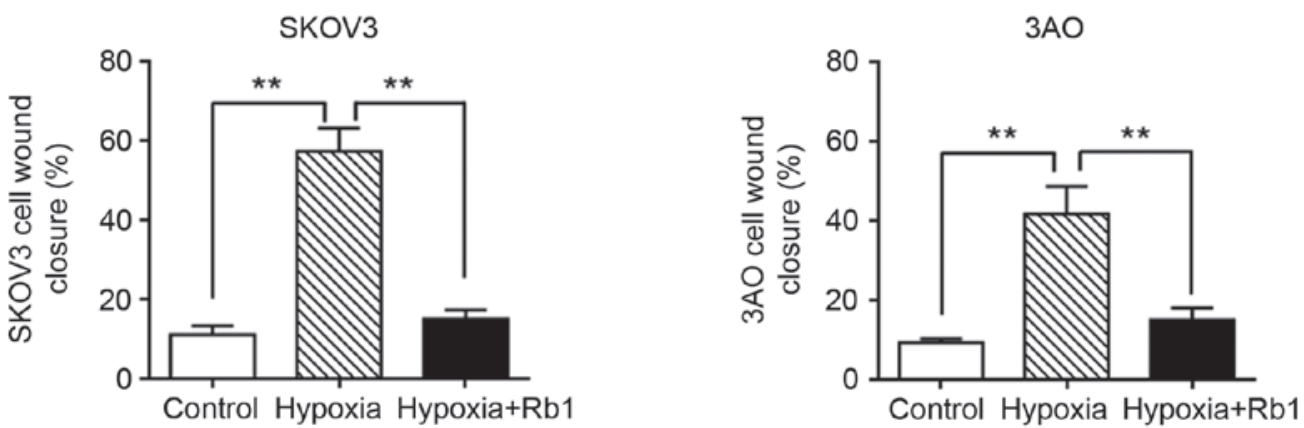

Figure 3. Wound healing assay of SKOV3 and 3AO cells. Scratch healing ability of (A) SKOV3 and (B) 3AO cells was significantly enhanced when cultured under hypoxic conditions for $24 \mathrm{~h}$, which was reversed by co-treatment with Rb1. Percentage of wound healing was calculated and statistically analyzed. ${ }^{* * *} \mathrm{P}<0.01$ as indicated.

was significantly enhanced by $24 \mathrm{~h}$ hypoxic incubation compared with the control $(\mathrm{P}<0.01)$, which was significantly weakened by $\mathrm{Rb} 1$ co-treatment $(\mathrm{P}<0.01)$. In vitro migration assays displayed similar results, $\mathrm{Rb} 1$ significantly reduced hypoxia-induced cell motility compared with the cells incubated under hypoxic conditions without the addition of Rb1 $(\mathrm{P}<0.01$; Fig. 4). Altogether, these results indicated that Rb1 may inhibit hypoxia-induced EMT in SKOV3 and 3AO cell lines.

Rb1 blocks hypoxia-induced EMT in SKOV3 cells by relieving the suppression of miR-25 on EP300. The mechanism mediating the inhibition by Rb1 on hypoxia-induced EMT in the SKOV3 cell line was then examined. Results demonstrated 


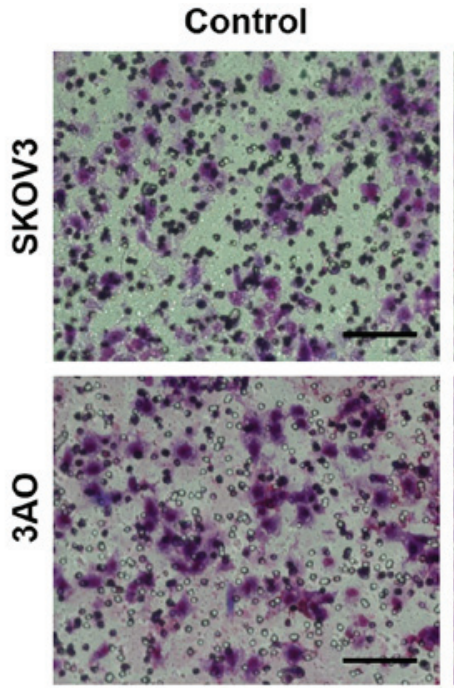

SKOV3

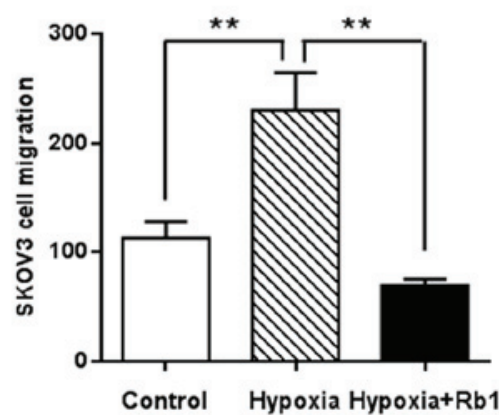

Ho24h
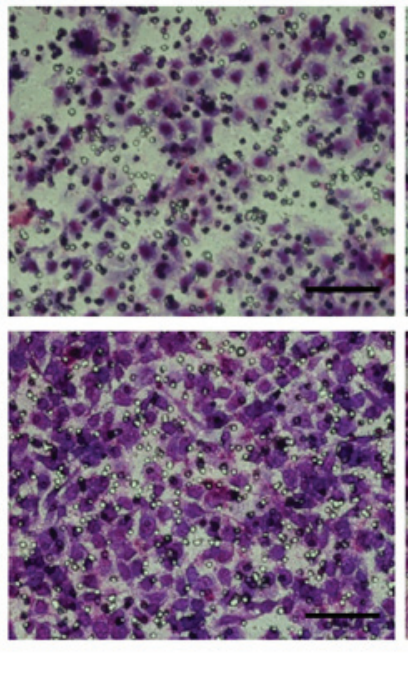
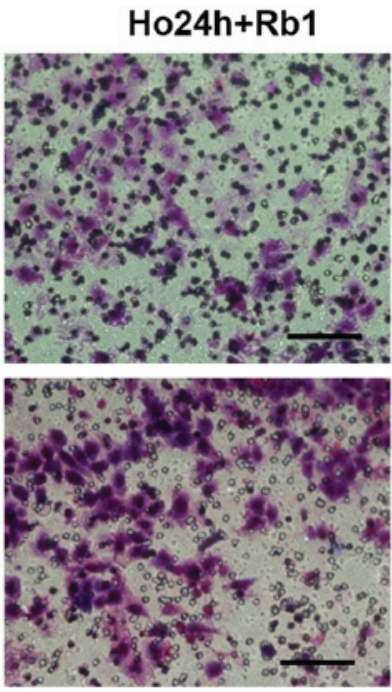

$3 \mathrm{AO}$

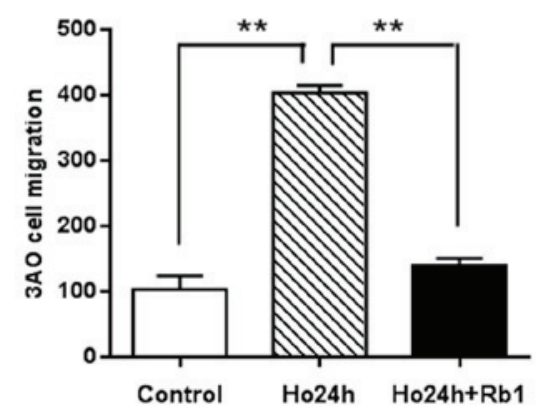

Figure 4. Cell mobility examined by in vitro migration assay. Compared with normoxic culture, Ho significantly increased motility of both SKOV3 and $3 \mathrm{AO}$ cell lines, which was reversed by co-treatment with Rb1. Cells that passed through the membrane were counted and used to represent in vitro cellular migration capacities. The assay was carried out in triplicate. Cells were stained using Giemsa staining. Scale bar, $100 \mu \mathrm{m} .{ }^{* *} \mathrm{P}<0.01$ as indicated. Ho, hypoxia.

that the expression of miR-25 was significantly increased during hypoxia-induced EMT in both SKOV3 and $3 \mathrm{AO}$ cells compared with control cells $(\mathrm{P}<0.01$; Fig. 5A). This increase in expression was significantly reversed by treatment with $\mathrm{Rb} 1$ $(\mathrm{P}<0.01$; Fig. 5A). In order to examine the role of miR-25 in the anti-EMT action of Rb1, miR-25 was overexpressed in SKOV3 cells by transfection with miR-25 mimics and detection of its effect on the role of Rb1 in hypoxia-induced EMT. RT-qPCR demonstrated that transfection with miR-25 mimics led to a significant increase ( $\sim 5$-fold) in the miR-25 expression level in SKOV3 cells compared with control cells ( $\mathrm{P}<0.01$; Fig. 5B). As detected by western blotting, exogenous overexpression of miR-25 itself reduced E-cadherin and elevated vimentin (Fig. 5C). miR-25 eliminated the capacity of Rb1 to block hypoxia-induced EMT (Fig. 5C). These results demonstrated that downregulation of miR-25 may be an important mechanism in the suppressive effect of Rb1 on hypoxia-induced EMT in ovarian cancer cells.

Following this, the potential target of miR-25 was identified. As EP300 is the transcriptional activator of E-cadherin (27), the changes in EP300 expression caused by miR-25 were examined. As demonstrated in Fig. 5D, overexpression of miR-25 repressed EP300 protein expression in SKOV3 cells. Furthermore, hypoxia reduced the protein expression level of EP300. Rb1 rescued the EP300 expression level under hypoxia, which was antagonized by exogenous miR-25 overexpression (Fig. 5E). These results suggested that
Rb1 blocked the hypoxia-induced EMT process in ovarian cancer cells via downregulation of miR-25, which contributed to the overexpression of E-cadherin transactivator EP300, thus increasing E-cadherin. Therefore, Rb1 may inhibit the hypoxia-induced EMT process in ovarian cancer cells through the miR-25/EP300/E-cadherin pathway (Fig. 6).

\section{Discussion}

EMT has been suggested as a predominant driver of poor survival in patients with ovarian carcinoma in a review summarizing gene expression array analyses, in which 108 of 154 genes selected by the authors were EMT-related (28). Efforts have been made to identify agents to block EMT in tumor therapy. EW-7195, a novel small molecule inhibitor specifically targeting TGF- $\beta$ type I receptor (ALK5) kinase, was demonstrated to decrease phosphorylated Smad 2 and the nuclear translocation of Smad 2 to inhibit transforming growth factor (TGF)- $\beta 1$-induced EMT in breast cancer cells (29). Anti-EMT therapeutics, particularly those with reduced cytotoxicity, would likely provide clinical benefits not shared by the commonly used genotoxic chemotherapy drugs that indiscriminately kill both normal and cancer cells, and result in severe side effects and even treatment failure (30). Therefore, identifying pharmacologically active ingredients from natural sources, such as Chinese herbs, to inhibit tumor metastasis could represent an attractive approach to anticancer drug discovery. 
A

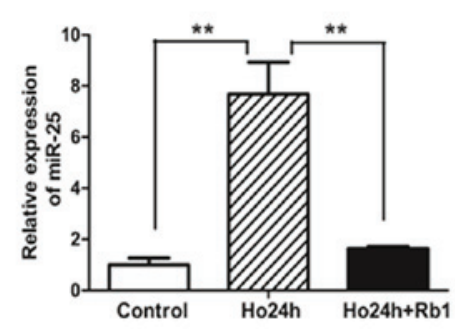

B

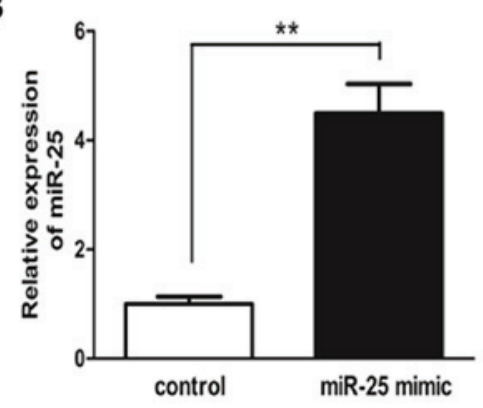

D

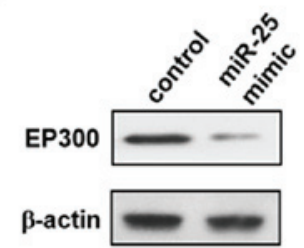

$3 A O$

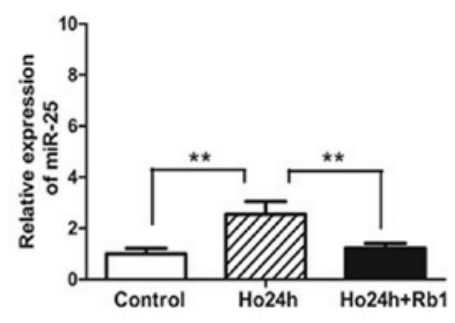

C

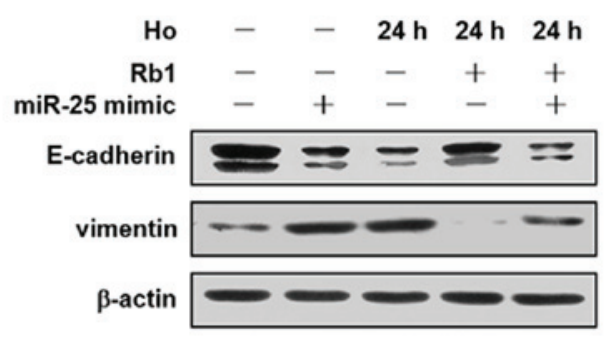

E

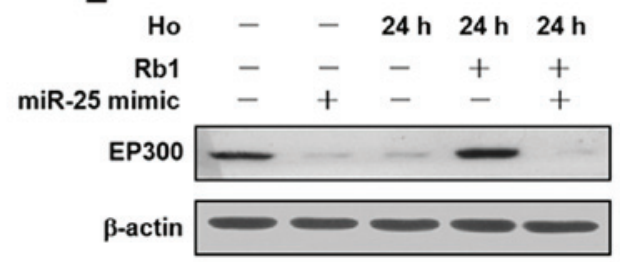

Figure 5. Rb1 inhibits miR-25 and releases its inhibition on EP300 to reverse hypoxia-induced EMT. (A) Rb1 negatively regulated miR-25. RT-qPCR results demonstrated that miR-25 level was increased in Ho SKOV3 and 3AO cells, which was recovered to the normal level by Rb1 treatment. Small nuclear RNA (Rnu6) was used as an internal control. (B) RT-qPCR results demonstrated that miR-25 mimic transfection led to an $\sim 5$-fold increase of miR-25 level in SKOV3 cells. (C) Overexpression of miR-25 repressed the anti-EMT action of Rb1 under the Ho condition. Western blotting results demonstrated that overexpression of miR-25 led to a decrease of E-cadherin and increase of vimentin in SKOV3 cells. Rb1 reversed Ho-induced E-cadherin reduction and vimentin elevation, which was blocked by overexpression of miR-25. (D) Western blotting demonstrated that miR-25 overexpression reduced EP300 protein level. (E) Western blotting results displayed that EP300 protein level was decreased by exogenous overexpression of miR-25 and Ho, while Rb1 slightly increased EP300 and antagonized the suppressive role of Ho and miR-25 on the expression of EP300. ${ }^{* *} \mathrm{P}<0.01$ as indicated. miR, microRNA; EMT, epithelial-mesenchymal transition; RT-qPCR, reverse transcription-quantitative polymerase chain reaction; Ho, hypoxia.

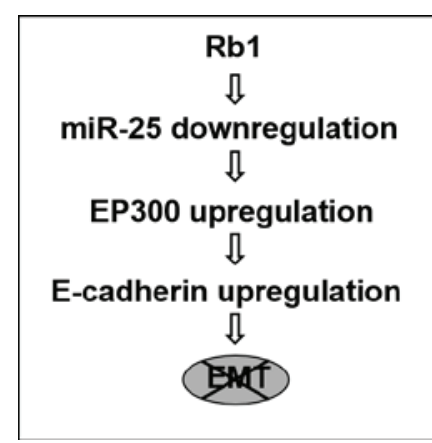

Figure 6. Schematic of anti-EMT pathway of Rb1. Based on the present study, $\mathrm{Rb} 1$ blocked hypoxia-induced EMT in ovarian cancer cells by releasing the suppression of miR-25 on EP300 to sustain E-cadherin expression. EMT, epithelial-mesenchymal transition; miR, microRNA.

Ginsenoside Rb1, the major bioactive component in $P$. ginseng, constitutes $0.47-0.82 \%$ of total $P$. ginseng root extract (31). The majority of published research on Rb1 has concentrated on the antioxidant and neovascularization effects of this component (32-35). The present study explored the anti-EMT effect of $\mathrm{Rb} 1$ in ovarian cancer. To the best of our knowledge, the present study is the first on the anti-neoplastic bioactivity of Rb1 in ovarian cancer. Being a natural herbal medicine, Rbl demonstrated no significant toxicity to human ovarian cancer cells. It inhibited EMT and migration of ovarian cancer cells in vitro. These results indicated that Rb1 may be a potential candidate for clinical application against ovarian cancer metastasis. At the same time, the possible underlying mechanism of Rbl's anti-EMT function was preliminarily explored. The present study demonstrated that $\mathrm{Rb} 1$ downregulated the expression of miR-25, leading to the overexpression of E-cadherin transcriptional activator EP300, thus increasing E-cadherin level and inhibiting the hypoxia-induced EMT process in ovarian cancer cells.

To date, published research has revealed the oncogenic involvement of miR-25 in apoptotic signaling $(14,36)$ and in cell invasion and migration $(37,38)$. A study by Smith et al (39) demonstrated that overexpression of miR-25 induced EMT in breast cancer through targeting the tumor-suppressive Smad7 protein, resulting in an increase in TGF- $\beta$ receptor 1 and downstream activation of TGF signaling. The same mechanism of miR-25 in inducing EMT was also reported in gastric 
cancer (40). A study by Wang et al (15) indicated that high miR-25 expression was significantly associated with advanced clinical stage and lymph node metastases in epithelial ovarian cancer. Notably, patients with a high expression of miR-25 tended to have shorter survival than patients with lower levels (10). Similarly, it was observed in the present study that miR-25 induced EMT in ovarian cancer cells. Rb1 suppressed the EMT process in ovarian cancer cells by downregulating miR-25. A study by Kwok et al (31) also identified that Rb1 may decrease the expression of miR-25 in human dermal fibroblasts, which is consistent with the results of the present. These findings indicated that miR-25 may be a potential target in ovarian cancer metastasis therapy.

EP300 is as a transcriptional cofactor and prototype histone acetyltransferase involved in regulating multiple cellular processes, including cell cycle regulation, proliferation, differentiation, apoptosis, DNA damage repair and adhesion, as well as embryonic development $(41,42)$. EP300 has been demonstrated to be a transcriptional activator of E-cadherin and to promote E-cadherin gene expression $(43,44)$. Furthermore, EP300 has been suggested to be the target gene of miR-25 in minimally transformed mammary epithelial cells (MTMEC) (27). In MTMEC cells, by targeting EP300, overexpressed miR-25 led to an EMT-like phenotypic characteristic change, including an increase in cell motility and invasion, as well as the ability to proliferate following treatment with doxorubicin (27). When EP300 was strongly expressed, the association between SNAIL expression and downregulation of E-cadherin was lost in colon carcinoma (45). Research has also demonstrated that loss of EP300 in HCT116 cells resulted in a potentially aggressive phenotype characterized by reduced adhesion and increased migration (46). Furthermore, several critical genes involved in these pathways are differentially expressed, suggesting that EP300 loss promoted EMT (46). Similarly, in the present study,EP300 was repressed during the hypoxia-induced EMT process in ovarian cancer cells, paralleled with E-cadherin downregulation. However, following treatment with Rb1, EP300 expression was recovered. Therefore, EP300 functioned in sustaining epithelial characteristics in cancer cells.

In conclusion, the results of the present study demonstrated that Rb1 could inhibit the hypoxia-induced EMT process in ovarian cancer cells through the miR-25/EP300/E-cadherin pathway. These results provided support for Rb1 acting as a useful drug candidate for ovarian cancer therapy, which warrants further research in the application of ginsenosides in cancer treatment.

\section{Acknowledgements}

The present study was supported by the National Natural Science Foundation of China (grant no. 30973429).

\section{References}

1. Siegel R, Ma J, Zou Z and Jemal A: Cancer statistics, 2014. CA Cancer J Clin 64: 9-29, 2014

2. Palma Flores C, Garcia-Vazquez R, Gallardo Rincón D, Ruiz-Garcia E, Astudillo de la Vega H, Marchat LA, Salinas Vera YM and Lopez-Camarillo C: MicroRNAs driving invasion and metastasis in ovarian cancer: Opportunities for translational medicine (Review). Int J Oncol 50: 1461-1476, 2017.
3. Cano A and Portillo F: An emerging role for class I bHLH E2-2 proteins in EMT regulation and tumor progression. Cell Adh Migr 4: 56-60, 2010.

4. Mallini P, Lennard T, Kirby J and Meeson A: Epithelialto-mesenchymal transition: What is the impact on breast cancer stem cells and drug resistance. Cancer Treat Rev 40: 341-348, 2014.

5. Li L and Li W: Epithelial-mesenchymal transition in human cancer: Comprehensive reprogramming of metabolism, epigenetics, and differentiation. Pharmacol Ther 150: 33-46, 2015.

6. Ye X and Weinberg RA: Epithelial-mesenchymal plasticity: A central regulator of cancer progression. Trends Cell Biol 25: 675-686, 2015.

7. Takai M, Terai Y, Kawaguchi H, Ashihara K, Fujiwara S, Tanaka T, Tsunetoh S, Tanaka Y, Sasaki H, Kanemura M, et al: The EMT (epithelial-mesenchymal-transition)-related protein expression indicates the metastatic status and prognosis in patients with ovarian cancer. J Ovarian Res 7: 76, 2014.

8. Bartel DP: MicroRNAs: Target recognition and regulatory functions. Cell 136: 215-233, 2009.

9. Kloosterman WP and Plasterk RH: The diverse functions of microRNAs in animal development and disease. Dev Cell 11: 441-450, 2006.

10. Chao A, Lin CY, Lee YS, Tsai CL, Wei PC, Hsueh S, Wu TI, Tsai CN, Wang CJ, Chao AS, et al: Regulation of ovarian cancer progression by microRNA-187 through targeting Disabled homolog-2. Oncogene 31: 764-775, 2012.

11. Chen J, Wang L, Matyunina LV, Hill CG and McDonald JF: Overexpression of miR-429 induces mesenchymal-to-epithelial transition (MET) in metastatic ovarian cancer cells. Gynecol Oncol 121: 200-205, 2011.

12. Petrocca F, Vecchione A and Croce CM: Emerging role of miR-106b-25/miR-17-92 clusters in the control of transforming growth factor beta signaling. Cancer Res 68: 8191-8194, 2008.

13. Savita U and Karunagaran D: MicroRNA-106b-25 cluster targets $\beta$-TRCP2, increases the expression of Snail and enhances cell migration and invasion in H1299 (non small cell lung cancer) cells. Biochem Biophys Res Commun 434: 841-847, 2013.

14. Zhang H, Zuo Z, Lu X, Wang L, Wang H and Zhu Z: MiR-25 regulates apoptosis by targeting Bim in human ovarian cancer. Oncol Rep 27: 594-598, 2012.

15. Wang X, Meng X, Li H, Liu W, Shen S and Gao Z: MicroRNA-25 expression level is an independent prognostic factor in epithelial ovarian cancer. Clin Transl Oncol 16: 954-958, 2014.

16. Vergara D, Valente CM, Tinelli A, Siciliano C, Lorusso V, Acierno R, Giovinazzo G, Santino A, Storelli C and Maffia M: Resveratrol inhibits the epidermal growth factor-induced epithelial mesenchymal transition in MCF-7 cells. Cancer Lett 310: 1-8, 2011.

17. Liao B, Newmark H and Zhou R: Neuroprotective effects of ginseng total saponin and ginsenosides Rb1 and Rg1 on spinal cord neurons in vitro. Exp Neurol 173: 224-234, 2002.

18. Nag SA, Qin JJ, Wang W, Wang MH, Wang H and Zhang R: Ginsenosides as anticancer agents: In vitro and in vivo activities, structure-activity relationships, and molecular mechanisms of action. Front Pharmacol 3: 25, 2012.

19. Jeong KJ, Kim GW and Chung SH: AMP-activated protein kinase: An emerging target for ginseng. J Ginseng Res 38: 83-88, 2014.

20. Choi JS, Chun KS, Kundu J and Kundu JK: Biochemical basis of cancer chemoprevention and/or chemotherapy with ginsenosides (Review). Int J Mol Med 32: 1227-1238, 2013.

21. Li J, Wei Q, Zuo GW, Xia J, You ZM, Li CL and Chen DL: Ginsenoside Rg1 induces apoptosis through inhibition of the EpoR-mediated JAK2/STAT5 signalling pathway in the TF-1/Epo human leukemia cell line. Asian Pac J Cancer Prev 15: 2453-2459, 2014.

22. Joh EH, Lee IA, Jung IH and Kim DH: Ginsenoside Rb1 and its metabolite compound K inhibit IRAK-1 activation-the key step of inflammation. Biochem Pharmacol 82: 278-286, 2011.

23. Luo T, Liu G, Ma H, Lu B, Xu H, Wang Y, Wu J, Ge P and Liang J: Inhibition of autophagy via activation of PI3K/Akt pathway contributes to the protection of ginsenoside Rb1 against neuronal death caused by ischemic insults. Int J Mol Sci 15: 15426-15442, 2014.

24. Wang CZ, Xie JT, Zhang B, Ni M, Fishbein A, Aung HH, Mehendale SR, Du W, He TC and Yuan CS: Chemopreventive effects of Panax notoginseng and its major constituents on SW480 human colorectal cancer cells. Int J Oncol 31: 1149-1156, 2007. 
25. Liu T, Zhao L, Zhang Y, Chen W, Liu D, Hou H, Ding L and Li X Ginsenoside 20(S)-Rg3 targets HIF-1 $\alpha$ to block hypoxia-induced epithelial-mesenchymal transition in ovarian cancer cells. PLoS One 9: e103887, 2014.

26. Livak KJ and Schmittgen TD: Analysis of relative gene expression data using real-time quantitative PCR and the 2(-Delta Delta C(T)) Method. Methods 25: 402-408, 2001.

27. Zhou Y, Hu Y, Yang M, Jat P, Li K, Lombardo Y, Xiong D, Coombes RC, Raguz S and Yague E: The miR-106b 25 cluster promotes bypass of doxorubicin-induced senescence and increase in motility and invasion by targeting the E-cadherin transcriptional activator EP300. Cell Death Differ 21: 462-474, 2014.

28. Yoshida S, Furukawa N, Haruta S, Tanase Y, Kanayama S, Noguchi T, Sakata M, Yamada Y, Oi H and Kobayashi H: Expression profiles of genes involved in poor prognosis of epithelial ovarian carcinoma: A review. Int J Gynecol Cancer 19: 992-997, 2009.

29. Park CY, Son JY, Jin CH, Nam JS, Kim DK and Sheen YY: EW-7195, a novel inhibitor of ALK5 kinase inhibits EMT and breast cancer metastasis to lung. Eur J Cancer 7: 2642-2653, 2011.

30. Liu Z, Qi S, Zhao X, Li M, Ding S, Lu J and Zhang H: Metformin inhibits $17 \beta$-estradiol-induced epithelial-to-mesenchymal transition via $\beta$ Klotho-related ERK1/2 signaling and AMPKo signaling in endometrial adenocarcinoma cells. Oncotarget 7: 21315-21331, 2016.

31. Kwok HH, Yue PY, Mak NK and Wong RN: Ginsenoside $R b_{1}$ induces type I collagen expression through peroxisome proliferator-activated receptor-delta. Biochem Pharmacol 84: 532-539, 2012.

32. Tan S, Zhou F, Li N, Dong Q, Zhang X, Ye X, Guo J, Chen B and Yu Z: Anti-fatigue effect of ginsenoside Rb1 on postoperative fatigue syndrome induced by major small intestinal resection in rat. Biol Pharm Bull 36: 1634-1639, 2013.

33. Liu Z, Chen J, Huang W, Zeng Z, Yang Y and Zhu B: Ginsenoside $\mathrm{Rb} 1$ protects rat retinal ganglion cells against hypoxia and oxidative stress. Mol Med Rep 8: 1397-1403, 2013.

34. Lin N, Cai DL, Jin D, Chen Y and Shi JJ: Ginseng panaxoside $\mathrm{Rb} 1$ reduces body weight in diet-induced obese mice. Cell Biochem Biophys 68: 189-194, 2014.

35. Liu D, Zhang H, Gu W, Liu Y and Zhang M: Neuroprotective effects of ginsenoside Rb1 on high glucose-induced neurotoxicity in primary cultured rat hippocampal neurons. PLoS One 8: e79399, 2013.
36. Razumilava N, Bronk SF, Smoot RL, Fingas CD, Werneburg NW, Roberts LR and Mott JL: miR-25 targets TNF-related apoptosis inducing ligand (TRAIL) death receptor-4 and promotes apoptosis resistance in cholangiocarcinoma. Hepatology 55: 465-475, 2012.

37. Xu X, Chen Z, Zhao X, Wang J, Ding D, Wang Z, Tan F, Tan X, Zhou F, Sun J, et al: MicroRNA-25 promotes cell migration and invasion in esophageal squamous cell carcinoma. Biochem Biophys Res Commun 421: 640-645, 2012.

38. Poliseno L, Salmena L, Riccardi L, Fornari A, Song MS Hobbs RM, Sportoletti P, Varmeh S, Egia A, Fedele G, et al: Identification of the miR-106b 25 microRNA cluster as a proto-oncogenic PTEN-targeting intron that cooperates with its host gene MCM7 in transformation. Sci Signal 3: ra29, 2010.

39. Smith AL, Iwanaga R, Drasin DJ, Micalizzi DS, Vartuli RL, Tan AC and Ford HL: The miR-106b-25 cluster targets Smad7, activates TGF- $\beta$ signaling, and induces EMT and tumor initiating cell characteristics downstream of Six1 in human breast cancer. Oncogene 31: 5162-5171, 2012.

40. Li F, Liu J and Li S: MicorRNA 106b 25 cluster and gastric cancer. Surg Oncol 22: e7-e10, 2013

41. Chan HM and La Thangue NB: p300/CBP proteins: HATs for transcriptional bridges and scaffolds. J Cell Sci 114: 2363-2373, 2001.

42. Iyer NG, Ozdag $\mathrm{H}$ and Caldas C: p300/CBP and cancer. Oncogene 23: 4225-4231, 2004.

43. Betel D, Wilson M, Gabow A, Marks DS and Sander C: The microRNA. org resource: Targets and expression. Nucleic Acids Res 36 (Database issue): D149-D153, 2008.

44. Liu YN, Lee WW, Wang CY, Chao TH, Chen Y and Chen JH: Regulatory mechanisms controlling human E-cadherin gene expression. Oncogene 24: 8277-8290, 2005.

45. Peña C, Garcia JM, Garcia V, Silva J, Dominguez G, Rodriguez R, Maximiano C, Garcia de Herreros A, Muñoz A and Bonilla F: The expression levels of the transcriptional regulators p300 and CtBP modulate the correlations between SNAIL, ZEB1, E-cadherin and vitamin D receptor in human colon carcinomas. Int J Cancer 119: 2098-2104, 2006.

46. Krubasik D, Iyer NG, English WR, Ahmed AA, Vias M, Roskelley C, Brenton JD, Caldas C and Murphy G: Absence of p300 induces cellular phenotypic changes characteristic of epithelial to mesenchyme transition. Br J Cancer 94: 1326-1332, 2006. 